\title{
Personalize Wayfinding Information for Fire Responders based on Virtual Reality Training Data
}

\author{
Jing Du \\ University of Florida \\ dujing82@gmail.com
}

\author{
Qi Wang \\ Northeastern University \\ q.wang@northeastern.edu
}

\author{
Yingzi Lin \\ Northeastern University \\ yi.lin@northeastern.edu
}

\author{
Changbum R Ahn \\ Texas A\&M University \\ ryanahn@tamu.edu
}

\begin{abstract}
Modern buildings with increasing complexity (e.g., more square feet, complicated indoor routes) can cause serious difficulties for first responders in emergency wayfinding. While real-time data collection and information analytics become easier in indoor wayfinding, a new challenge has arisen: cognitive overload due to information redundancy. Standardized and universal spatial information systems are still widely used, ignoring first responders' individual difference in information intake. This paper proposes the theoretical framework of a spatial information systems for first responders, which reflects their individual difference in information preference and helps reduce the cognitive load in line of duty. The proposed method includes the use of Virtual Reality (VR) to simulate real world buildings, and the modeling of first responders' reactions to different information formats and contents in simulated wayfinding tasks. This work is expected to set a foundation of future spatial information system that correctly and effectively responds to first responders' needs.
\end{abstract}

\section{Introduction}

Buildings are becoming unprecedentedly complex, e.g. more square feet, complicated indoor routes, an increasing usage of temporary furniture and decors, along with the fast development of building information technologies. By 2020 , about $20 \%$ of U.S. buildings can be classified as "smart" with 50 billion connected devices forming clusters of Internet of Things (IoT) [1] These new sensing devices and technologies will generate a large amount of real-time informatics in built environments. The way a building's spatial information is presented is becoming more diverse, mixing signage (e.g., directional, identification, information, warning), text instructions, maps (2D, 3D, interactive), tactile and haptic guidance, sound, verbal guidance, colors, landmark markers, and lighting, etc. [2]. While real-time data collection and information analytics become easier in complex building wayfinding, a new challenge has arisen: cognitive overload due to information redundancy. Too much information can lead to cognitive overload [3], causing decreased task performance [4] and/or prejudices in decisions such as stereotyping, i.e., relying on personal experiment instead of facts [5]. In emergency wayfinding, first responders often need to build an accurate spatial working memory of unfamiliar spaces in a timely manner, requiring an intensive retention and processing of received spatial information (such as maps or verbal instructions) [6]. Yet the well-proven Miller's Law shows that humans are extremely limited in information processing capacity [7]. Such an apparent gap between the enormous information processing need and the limited processing capacity creates a potentially fatal situation in emergency response for both the rescuers and rescuees.

Each person is unique in how they process information intake: some may be easily burdened by visuospatial information, while others may be reluctant to verbal instructions [8]. The information-taking behavior of the same person may also change dramatically in different situations (e.g., leaning to visuospatial information in extremely emotional events) [9]. This indicates that the method of controlling information-induced cognitive load of first responders in emergencies varies person by person, case by case. Despite the cognitive variance, there is a lack of an effective platform to allow first responders to fully exhibit their behaviors, record their cognitive patterns, detect their variances, and build their profiles to guide their actions during emergencies.

The objective of our study is to introduce the theoretical framework of a personalized spatial information system (PSIS) for first responders in emergency indoor wayfinding. PSIS dynamically tailors the way spatial information is presented based on realtime cognitive load of first responders at the individual level, and reflects individual difference in spatial information intake. PSIS is expected to improve balancing of the cognitive load and achieve cognitive resilience of emergency first responders. 


\section{Literature Review}

\subsection{Personalized information systems}

With the and machine learning technologies, personalized information systems (PIS) have become popular in our daily lives and are affecting average population's behaviors. PIS are information communication technologies that consider and adapt to personalized behavior, adjusting to the preferences and needs of their users [10], and include applications such as personalized Google search results [11], Facebook feeds [12], and news alerts [13]. These systems can learn individual behaviors and automatically filter out the least valued information for the users.

However, universal spatial information systems that don't differentiate individual differences in information demand are still heavily used in public wayfinding, such as maps, signage, and textural instructions, etc. Government agencies still advocate standardized design principles for wayfinding information [14-16], as well as standardized information and workflows in emergency response [17], which may cause an imbalance between information supply and demand in wayfinding. To address this imbalance, the indoor navigation literature has started to investigate the personalization of spatial information. The use of RFID [18], indoor localization [19] and AR technologies [20] have greatly improved situational awareness; by providing accurate location information, they are able tohelp reduce the amount of information to be processed. This research aims to complement these personalization studies by investigating how real-time cognitive load can be used as a baseline for dynamic adaption of information presentation methods by taking individual differences in information processing capacity into consideration [21].

\subsection{Cognitive load and information intake}

Cognitive load is driven by various mental activities and influences decision-making in distinct ways [2228]. Cognitive load theory (CLT) [29-32] divides the overall cognitive load into three main components: intrinsic cognitive load (related to the complexity of tasks), extraneous cognitive load (affected by how information is presented) and germane cognitive load (devoted to construction of schemas - permanent knowledge about patterns). Baddeley and colleagues proposed a dual-coding theory to describe the internal structure of extraneous cognitive load in relation to the working memory of information processing $[25,26,28$, 33-36]. They found that there exists "dual channels" in human cognition, where different mental activities are activated when people are processing two distinct categories of information: phonological information (i.e., auditory verbal information or visually presented language) and visuospatial information (i.e., the visually presented information about objects and space). In addition, a central executive cognitive process also takes place to bind information into coherent episodes, shift between tasks or retrieval strategies, and select between attention and inhibition [37]. Mayer et al. [38] further discovered that information relates to various mental constructs including sensory (which receives stimuli and stores it for a very short time), working (where we actively process information to create mental constructs), and long-term (the repository of all things learned) memories [38].

The heterogeneous structure of cognitive load has been empirically validated by recent neurobiological evidence. For example, neuroimaging studies found that phonological information often triggers Broca's area (involved in speech production) in addition to supplementary and premotor areas (involved in movement) in frontal cortex cognitive [39]. Neural areas related to spatial or object storage are activated in pursuit of the visuospatial information such as the bilateral intraparietal sulcus region [40]. The dorsolateral prefrontal cortex (DLPFC) of brains is always activated when central executive process happens [37]. Electroencephalogram (EEG) studies also identified distinguishable patterns of various brain waves corresponding to different types of cognitive load $[41,42]$.

The literature has recognized the difficulty of measuring cognitive load caused by different information. As a result, navigation and driver behavior literature tends to investigate wayfinding-related cognitive load as a single entity [43-47]. Indicators such as task performance (e.g., errors) and psychometrics (e.g., surveys) are typically used to measure cognitive load in these studies [4, 42, 48], and although these are important measurements to consider, the lagging nature of the indicators are insufficient to support a real-time monitoring and control mechanism of cognitive load that attempts to distinguish different types of cognitive load (e.g., visual and verbal-driven).

\section{Proposed Personalized Spatial Information System (PSIS) for Emergency Wayfinding}

The challenge to build a personalized spatial information system, i.e., PSIS, for first responder wayfinding does not only lay at the information filtering and presenting. A tailored information system can respond to a first responder's needs correctly and effectively only if the profile is established based on 
historical data. An information system without a robust database and analytical system to store, process, and analyze first responders' actions cannot support an individual's training or actions during emergencies even though it is equipped with the most advanced visualization technologies. It must address the following questions:

1) Can we build a database of first responders' information-taking behaviors during their training and/or operations?

2) How can wayfinding UI be tailored to adapt to the established information-taking database of first responders?

We focus on the test of PSIS, to control the real-time cognitive load of first responders in emergency wayfinding. To establish the basis of spatial information personalization, we propose using Information Personality, a cognitive profile of information-taking preference and behavioral patterns at the individual level. PSIS dynamically and automatically customizes UI to each individual first responder based on their unique Information Personality. Information Personality can be tracked by quantifying individual reactions to different types, quantities, and display methods of information during virtual reality (VR) training. Information Personality data of individual first responders can be integrated as a necessary part of their personal files. Just as personality can be used to model behavioral patterns of a person, the unique information-taking patterns captured by Information Personality also exhibit individual differences and help tailor UI to reduce their cognitive load. Fig.1 illustrates the concept map of PSIS. The rest of this section will describe details of PSIS.

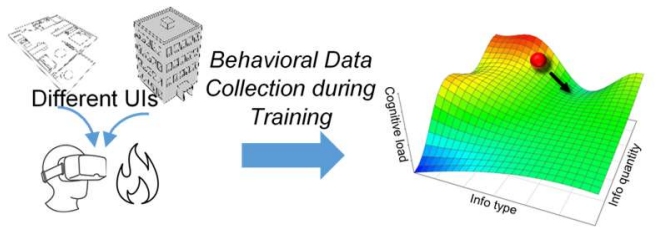

Step1: First responder Step 2: Develop info personality model VR Training

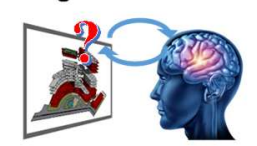
that reflects repetitive pattern of info taking

Step 3: Dynamically adjust UI based on info personality under different levels of cognitive load

Fig.1 Concept map of PSIS and methods

\subsection{VR training to collect raw data about information personality}

The first component of PSIS is a VR training platform that collects personal data of spatial information intake. The key step is the development of VR model modules that accurately reconstruct various emergency scenarios for training purposes. We have developed a VR fire training platform (Fig.2) to include valuable field knowledge, such as the fact that firefighters tend to prioritize actions based on the color of smoke (black or white).

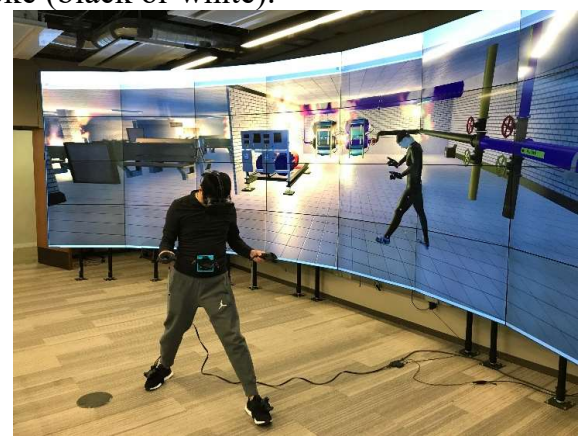

Fig. 2 A VR simulation platform for information personality data collection

To examine how first responders react to the perceived spatial information, we need to collect realtime cognitive load (CL) during training. PSIS highlights the importance of differentiating different types of CL as a multidimensional construct. Based on the Cognitive Load Theory and Baddeley's model of working memory [26], PSIS measures cognitive load as a summation of intrinsic CL (related to task difficulty) and extraneous CL (related to received information). Although psychometrics-based cognitive load measurements (e.g., NASA TLX Survey (Task Load Test [49]) have been well-validated, they have rarely been used as a working instrument in real-time monitoring. To overcome this challenge, PSIS uses a comprehensive CL index based on a variety of psychological and neurobiological metrics, including brain activity, physiological, and ergonomic indicators. Based on a comprehensive literature review, the following (12) metrics for real-time CL measurement metrics are used as listed in Table 1.

Table 1. Real-time cognitive load measurement metrics

Real-Time Cognitive Load Metrics

1) Electroencephalogram (EEG): Certain EEG signals relate to extraneous cognitive load (e.g., cognitive load induced by verbal, visual, textural info)

2)Tapping Frequency (TF): The tapping frequency of feet and fingers

3) Gait Features (GF): Gait variability such as change to stride length

4) Pupillary Dilation (PD): Task-invoked pupillary response in diameter change

5) Eye Movement Frequency (EMF): Pixels moved per second

6)Eye Blink Rate (EBR): Eye blinks per minute 
7) Electrocardiogram (ECG): The electrical activity of the heart

8) Respiratory (RES): Total volume and rate

9) Galvanic Skin Response (GSR)

10) Heat Flux Rate (HFR): Rate of skin heat transfer

11)Electromyography (EMG): Muscle activities

12)Heart Rate-Blood Pressure Product (RPP): Heart rate and systolic blood pressure

PSIS raw data collection also includes the wayfinding performance of trainees. Navigation literature has proposed a variety of wayfinding performance metrics (e.g., [50]); however, many of these metrics are size-driven, i.e., dependent upon the search space size. For example, total wayfinding time in a larger area is naturally longer than that in a smaller area, even though the cognitive load in two scenarios could be similar. Scale-variant measurements cannot explain the relationship between cognitive load and wayfinding performance. To establish the baseline between cognitive load and wayfinding performance, PSIS uses scale-free wayfinding performance metrics (Table 2). All data will be collected or converted into JSON format for its easiness of application and storage.

Table 2. Scale-free wayfinding performance metrics

1) Speed or Average Time ( $\mathrm{m} / \mathrm{s})$ : The average travel speed between two waypoints

2)PAO (\% above optimal path, i.e. the theoretically shortest path): The distance that was travelled additionally to the optimal route

3)Directional Error $\left({ }^{\circ}\right)$ : Average deviation from the optimal direction in open areas

4)Wrong Turns (n): Average number of wrong turns at critical intersections

5)Intentional Stops (n): Average number of intentional stops for wayfinding

6)Intentional Stop Time (s): Average intentional stop time for wayfinding

7)Backtracking (n): Average number of backtracking

8) Reported Lost (n): Average number of reported lost by subjects

9) Verbal Comments: Subjects' verbal comments about difficulty, landmarks etc.

10) Sketch Map Accuracy: Statistical measure of the accuracy of memorized map

\subsection{Information personality modeling}

The second component of PSIS is the information personality models and corresponding optimization algorithm. A controlled Sternberg working memory test [51] will be used to (1) standardize the modeling of information personality and (2) collect continuous data about the complete picture of the cognitive impacts of spatial information of different kinds. Participating first responders are asked to finish a dual task: 1) Primary task - Sternberg working memory test. As illustrated in Fig. 3, subjects are asked to take a Sternberg working memory test; The test starts with the presentation of a list of spatial information items to memorize (encoding or learning period) and then has (1) a memory retention period during which the subject must maintain the list of items in memory and (2) a retrieval period in which the subject will answer if a given item appeared. Between two sessions is a short period of fixation phase. The Sternberg test is repeated 150 times for each subject, and the working memory accuracy will be used as a direct indicator of cognitive load as suggested by the literature [24, 52-54]. 2) Secondary task - Search task. As shown in Fig.4, while the subject is taking the Sternberg test, he or she is also asked to search for a missing person, following the given instructions. While the subject is performing the Sternberg test, the cognitive load and attention pattern data is documented to evaluate the impacts of information.

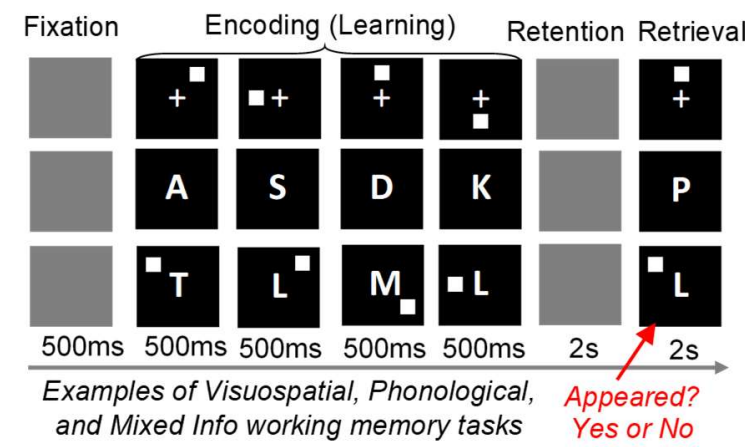

Fig. 3 Sternberg working memory test (repetitive). Test subjects need to memorize the info provided and answer if it appeared based on memory

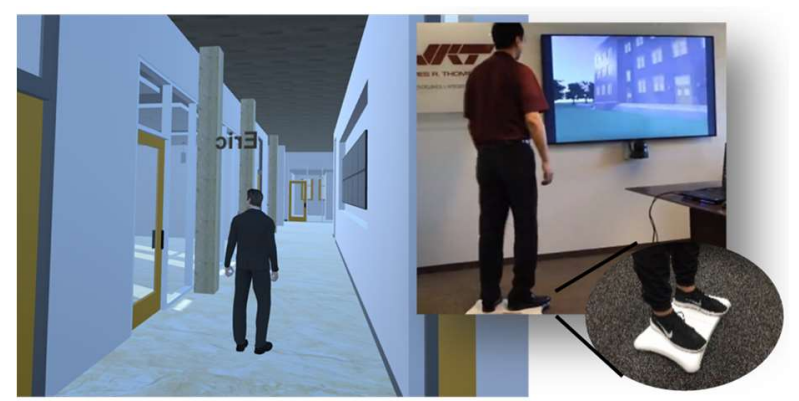

Fig.4 Test subjects are also asked to search for a person. We programmed Wii Balance Board for avatar control by leaning and walking on the board.

Once the raw data is collected, PSIS will develop a comprehensive index of Information Personality that captures the individual differences in informationtaking. According to the multimedia learning theory [12], PSIS focuses on modeling Information Personality 
from two dimensions: information quantity and information type. The former one refers to how much meaningful content is delivered in a message (i,e., semantic contents), while the latter one shows if the information is more abstractly displayed (such as textual instructions) or more visually displayed (such as an interactive map). Finally, a continuous landscape of Information Personality is established to predict (in general) how the cognitive load of a first responder changes in different scenarios. Fig.5 illustrates two hypothetical cases of information personality models. As discussed earlier, information personality model indicates personalized ways of reducing cognitive load: at the same level of cognitive load, the best strategy to reduce Eric's cognitive load is to switch to a different information type, which represents the steepest gradient decent to lower cognitive load; while for Ryan, it is better to reduce the quantity. PSIS therefore applies a UI adaption algorithm based on each first responder's unique Information Personality. At any given level of cognitive load, a gradient descent algorithm will be applied to find the most effective way of reducing cognitive load by adjusting the specific UI display design
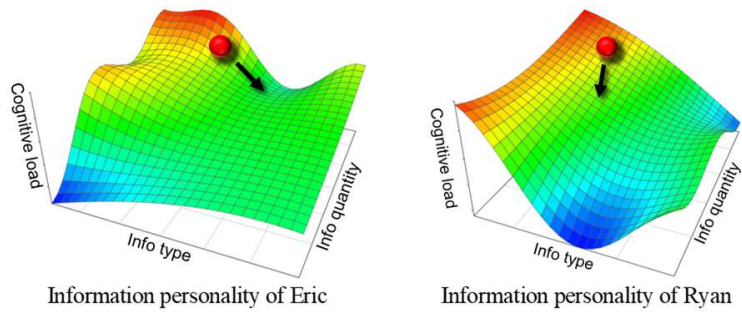

Fig.5 Two hypothetical examples of Information Personality

\subsection{Adaptive UI based in information personality models}

Ultimately, PSIS focuses on the adaption of spatial information based on the individual information personality models to reduce cognitive load in emergency wayfinding. We have examined the relevance of different building information in the context of emergency wayfinding, as well as the choices of the UI designs recommended by NIST standards. In the past 10 years, NIST has published a series of standards or reports on the public safety information systems in the context of building search and rescue, such as [55-57]. Based on a comprehensive literature review of NIST standards and relevant publications (e.g., NEMA SB30 [58]), PSIS builds the wayfinding UI based on spatial information as illustrated in Fig. 6 .

Then PSIS will adjust the UI from a series of possible solutions. In spite of the specific differences, PSIS shows wayfinding information at three levels:
- Level 1 - Permanent Display, i.e., important spatial info that is always displayed on the periphery;

- Level 2 - Adaptive Display, i.e., context-driven info adjusted by the real time cognitive load;

- Level 3 - Salient Display, i.e., alert info that appears on the center of the display when needed.

All the information will be displayed in four types of format according to the Baddeley's working memory theory [13], including visual info (e.g., signage, maps, pictures, colors, lights etc.), textual (e.g., written or verbal instructions), sensory info (e.g., haptic) and mixed info (mixed use of two or more different types). The envisioned PSIS UI is illustrated in Fig.7.

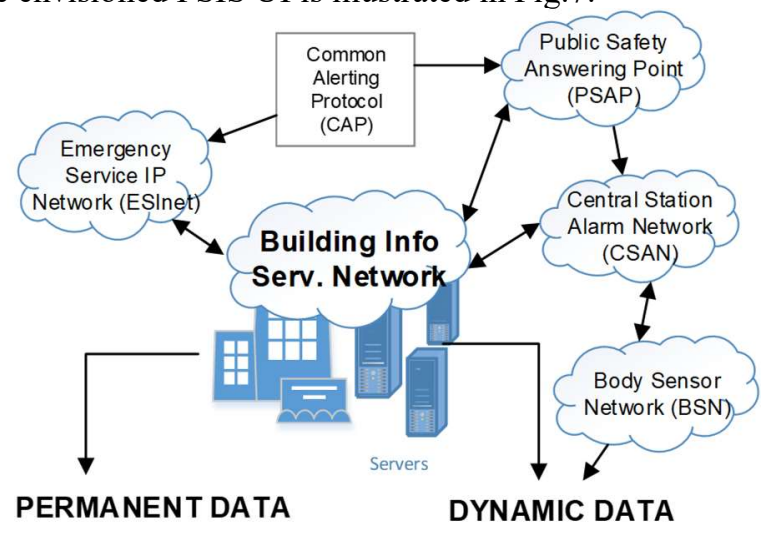

Building Metadata

(e.g., use, age, contacts)

Spatial Data

(e.g., floor plan, 3D model, exits)

Fire Response Features

(e.g.,water access, areas of refuge, utility shutoffs)

Emergency Serv. Features

(e.g.,hazardous materials)

Site Features

(e.g.hydrants, egress path)

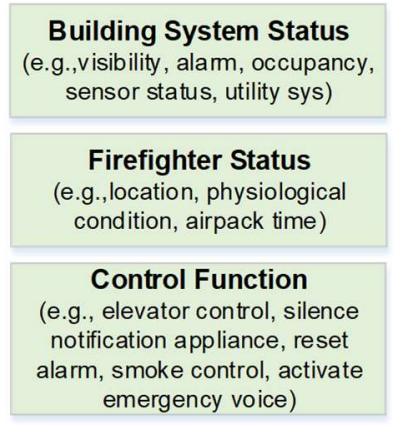

Fig.6 Relevant spatial information in emergency wayfinding

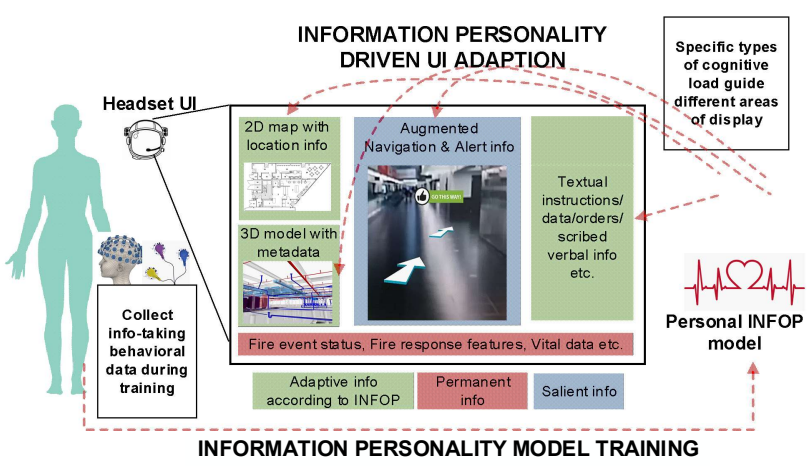

Fig. 7 Illustration of PSIS UI Design 
Driven by the learned Information Personality model of individual first responders and the real-time measure of different types of cognitive load, the algorithms employed by PSIS will determine: (1) What information content should be presented (semantics); (2) When the information should be presented (time); and (3) How the information should be presented (syntactical issue). Percentage of standardized and personalized information will also be determined based on personal preference. Information is categorized into three levels as discussed earlier, including permanent information, adaptive information, and salient information; Adaptive information will be customized based on Information Personality. Following the dualcoding theory [26], PSIS maps cognitive load as a multidimensional constructs, including phonological process load (i.e., auditory verbal information or visually presented language), visuospatial sketchpad load (i.e., the visually presented information about objects and space), and central executive load (i.e., cognitive load related to binding information into coherent episodes, shifting between tasks or retrieval strategies, and selecting between attention and inhibition) [37]. Instead of adjusting the UI display as a whole piece, the specific type of cognitive load will be monitored to adjust only the corresponding areas/types of information in the UI.

\section{Preliminary Findings}

In order to test the key concepts of the proposed PSIS, i.e., the influence of information on spatial cognition, we performed a pilot study at Texas A\&M University. Test subjects $(\mathrm{n}=54)$ were asked to perform a spatial cognition experiment with two sessions: 1) review session: test subjects were asked to review the floorplan information of Francis Hall (a building at Texas A\&M University) for five minutes. Depending on what information was given, test subjects were grouped into 2D group (2D maps were given; $n=18$ ), 3D group (computer 3D models were given; $\mathrm{n}=18$ ), and VR group (VR models were given; $\mathrm{n}=18$ ); 2) inspection session: the test subjects were asked to inspect the real building to identify any discrepancy between the reviewed floorplan information and the real building. A total of 28 points of interest (POIs) were used in the inspection session. The inspection results of the test subjects were used as an indicator of their spatial cognition. Their gaze focus and navigation trajectories in the review sessions were also documented using Tobii eye tracker and motion trackers. Fig.8 illustrates the aggregated attention and navigation patterns. Green lines indicate the gaze focus, and red lines indicate the navigation trajectories in the space.
The preliminary result suggests that the attention patterns and spatial cognition performance of subjects were substantially affected by the received spatial information.

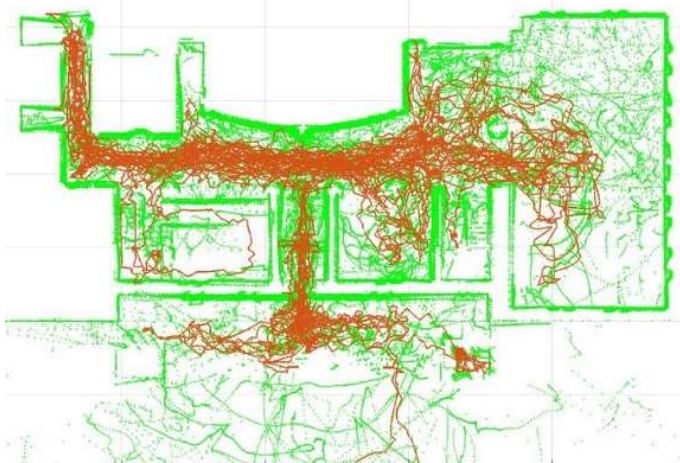

Fig.8 Documented gaze and navigation trajectories (green: gaze fixation; red: navigation trajectory)

Fig. 9 indicates that different information formats (2D map, 3D model, and VR model) significantly affected the distribution of gaze fixation time of the test subjects on the 28 POIs during the review session, which later resulted in different spatial cognition performance. More specifically, we discovered that 2D information results in a narrower distribution compared to 3D and VR group, suggesting that test subjects tended to move their eyeballs much faster when reviewing $2 \mathrm{D}$ maps (Fig.9, left). A more interesting finding pertains to the relationship between gaze fixation time and the likelihood of finding the discrepancies. As shown in Fig.9 (right), the likelihood of finding the discrepancies increases as the gaze fixation time increases during the review session; nonetheless the increasing slops across three groups are very different.
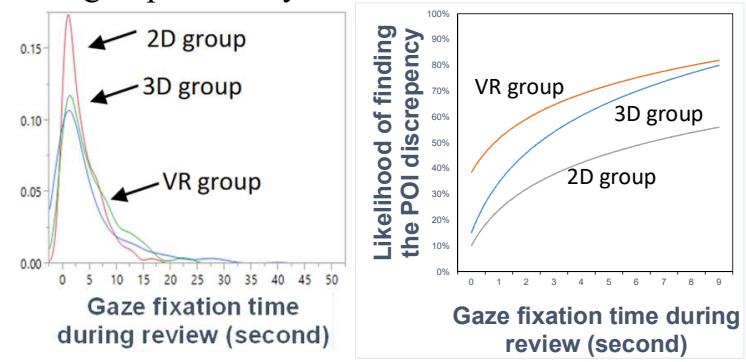

Fig.9 Spatial information affects gaze fixation time distribution and spatial cognition

We have also tested part of the cognitive load metrics in a pilot study. As illustrated in Fig.10, we have successfully identified brain wave patterns (EEG) related to the increased cognitive load in the Sternberg experiments. More specifically, 3D and VR seem to trigger higher Alpha wave levels, suggesting higher mental load in forming the working memory (Fig.10). One of the features of the proposed system is to train and 
study first responders' wayfinding behaviors based on a digital replica of real-world built environments. It contributes to the Smart City Digital Twins by providing an automated way of collecting human wayfinding behaviors and thus, it enables analytical capacities pertaining to the residents of Smart City.

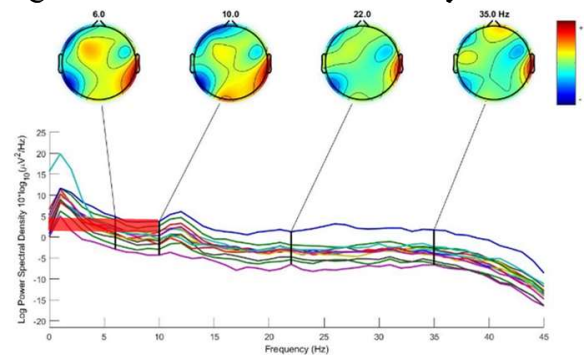

a. Alpha wave spectrogram when reviewing 2D

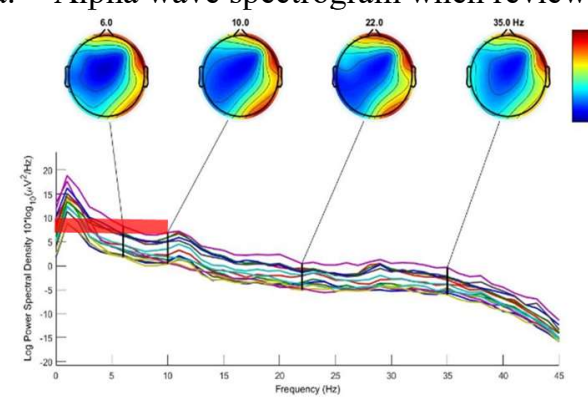

b. Alpha wave spectrogram when reviewing 3D

Fig.10 EEG result of different information formats

\section{Discussion}

The proposed PSIS and corresponding methods are expected to advance the knowledge and technology of wayfinding systems for first responders in two ways. First, PSIS highlights a personalized UI solution based on the individual information-taking characteristics, or Information Personality. The existing literature has recognized the importance of optimizing UI to control the cognitive load of first responders [59-62]. However, meantime most literature only focuses on giving generalized UI design recommendations rather than providing personalized solutions. The assumption is that certain principals of UI design would work well to a broad range of population [62]. Yet this assumption is questionable as cognitive science literature has already discovered that individual level difference in information taking is a major contributor to the varying task performance [8]. PSIS employs sensing technologies (e.g., mobile EEG and body carried sensors) and algorithms to track and model Information Personality characteristics of individual first responders. The Information Personality models will then be used to guide the dynamic change of UI in line of duty. When the cognitive load of a first responder is sensed to be high, the system will adjust the amount, content and format of UI to reduce the cognitive load, according to the patterns captured by her Information Personality model. This personalized UI solution will transform the UI technologies into an adaptive framework.

Second, PSIS integrates behavioral data collection and modeling during the VR training. There have been a variety of successful efforts in bringing VR technologies to emergency response training. Representative examples include FLAIM [63], E2i Creative Studio [64], and ADMS-FIRE [65]. These efforts are effective in training first responders as they can simulate the hazardous situations hardly reproducible in real world and bring immersive experience to the first responders. PSIS moves a step further by adding personal behavioral data collection and study function in the VR training system. Equipped with the new sensing technologies, personal data of first responders that is used to be hard to access will be collected with the daily training. Unlike existing methods of psychometrics surveys, collecting personal data with training will help trigger natural (and realistic) reactions of first responders as the participants will not be aware of it. In addition, collecting behavioral data with daily training will be very cost effective as it does not require expensive experiment setups as often seen in designated psychological experiments.

\section{Conclusions}

This paper introduces the theoretical framework of a personalized spatial information system, i.e., PSIS, for emergency wayfinding of first responders. The core components of PSIS including a VR training platform that collects personal data of information preference during the training, the modeling and optimization algorithm of information personality (repetitive pattern of information intake behaviors) of individual first responders, and the adaptive UI design based on the real time cognitive load and information personality models. By dynamically adjusting the contents and formats of presented spatial information, PSIS is expected to reduce the real time cognitive load of first responders in line of duty, while still maintaining an effective wayfinding.

This study is expected to help transform the emergency risk communication theory from a focus on a universal and standardized information flow to a more personalized process, driven by individual differences in information processing capacity. Information filtering theory [66] and cognitive load theory [29] both point that information overload is a significant driver of errors and mistakes in this information-rich era. Although interactive information systems have been proposed by the emergency response literature, there is still an urgent need for a cognitive-load driven method. 
This study will also help settle the argument whether more flexibility and interactivity in modern emergency information systems (such as interactive maps) could benefit professional responders and general public, as well as what should be the ratio of general and personalized information in practical implementation. Empirical experiments will provide a quantitative answer to whether allowing information choice decisions may increase one's cognitive load. Answering these questions are critical to the development of the principles of the next generation emergency information systems. The future agenda of this study will focus on the validation of the proposed Personalized Spatial Information System (PSIS) in improving the effectiveness and efficiency of emergency wayfinding, as well as the added health benefits to the first

[1] Memoori, "Big Data for Smart Buildings 2015 to 2020," 2015, Available: https://www.memoori.com/portfolio/bigdata-smart-buildings-2015-2020/.

[2] C. Calori and D. Vanden-Eynden, Signage and wayfinding design: a complete guide to creating environmental graphic design systems. John Wiley \& Sons, 2015.

[3] M. J. Eppler and J. Mengis, "The concept of information overload: A review of literature from organization science, accounting, marketing, MIS, and related disciplines," The information society, vol. 20, no. 5, pp. 325 344, 2004.

[4] J. Sweller, "Cognitive load during problem solving: Effects on learning," Cognitive science, vol. 12, no. 2, pp. 257-285, 1988.

[5] M. R. Banaji and A. G. Greenwald, "Implicit stereotyping and prejudice," in The psychology of prejudice: The Ontario symposium, 1994, vol. 7, pp. 55-76.

[6] R. H. Logie, Visuo-spatial working memory. Psychology Press, 2014.

[7] G. A. Miller, "The magical number seven, plus or minus two: Some limits on our capacity for processing information," Psychological review, vol. 63, no. 2, p. 81, 1956.

[8] C. Cornoldi, A. Cortesi, and D. Preti, "Individual differences in the capacity limitations of visuospatial short-term memory: Research on sighted and totally congenitally blind people," Memory \& Cognition, vol. 19, no. 5, pp. 459-468, 1991.

[9] E. A. Holmes and C. Bourne, "Inducing and modulating intrusive emotional memories: A review of the trauma film paradigm," Acta responders. We also expect a more thorough experiment to reveal what types of information are more suitable to be presented in $2 \mathrm{D}, 3 \mathrm{D}$, or VR environments. We will also focus on the studying the existing data standards relevant to firefighting, including NFPA 950.

\section{Acknowledgements}

This material is supported by the National Science Foundation (NSF) under Grant Numbers 1761459 and 1761950, as well as National Institute of Standards and Technology (NIST) under Grant Number 60NANB18D152.

\section{References}

Psychologica, vol. 127, no. 3, pp. 553-566, 2008.

[10] P. W. Foltz and S. T. Dumais, "Personalized information delivery: An analysis of information filtering methods," Communications of the ACM, vol. 35 , no. 12 , pp. 51-60, 1992.

[11] M. Speretta and S. Gauch, "Personalized search based on user search histories," in Web Intelligence, 2005. Proceedings. The 2005 IEEE/WIC/ACM International Conference on, 2005, pp. 622-628: IEEE.

[12] A. G. Bosworth, C. Cox, R. Sanghvi, T. S. Ramakrishnan, and A. D'angelo, "Generating a feed of stories personalized for members of a social network," ed: Google Patents, 2010.

[13] M. Eichstaedt, A. P. Patel, Q. Lu, U. Manber, and K. Rudkin, "System and method for personalized information filtering and alert generation," ed: Google Patents, 2002.

[14] R. Apelt, J. Crawford, and D. J. Hogan, Wayfinding design guidelines. $\mathrm{CRC}$ for Construction Innovation, 2007.

[15] T. f. L. TFL. (2017). Wayfinding. Available: https://tfl.gov.uk/info-for/urban-planningand-construction/wayfinding

[16] L. T. A. o. S. LTA. (2017). Guidline for Adequate Provision of Wayfinding Signage within Developments. Available: https://www.lta.gov.sg/content/dam/ltaweb/c orp/GreenTransport/2016/Guide $\% 20$ for $\% 20$ Wayfinding\%20Signage.pdf

[17] FEMA, "National Urban Search \& Resecue Response System: Rescue Field Operations Guide," 2006, Available: https://www.fema.gov/pdf/emergency/usr/us r 23 20080205_rog.pdf.

[18] Y.-J. Chang, C.-N. Chen, L.-D. Chou, and T.Y. Wang, "A novel indoor wayfinding 
system based on passive RFID for individuals with cognitive impairments," in Pervasive Computing Technologies for Healthcare, 2008. PervasiveHealth 2008. Second International Conference on, 2008, pp. 108-111: IEEE.

[19] J. Torres-Solis, T. H. Falk, and T. Chau, "A review of indoor localization technologies: towards navigational assistance for topographical disorientation," in Ambient Intelligence: InTech, 2010.

[20] B. F. Goldiez, A. M. Ahmad, and P. A. Hancock, "Effects of augmented reality display settings on human wayfinding performance," IEEE Transactions on Systems, Man, and Cybernetics, Part C (Applications and Reviews), vol. 37, no. 5, pp. 839-845, 2007.

[21] D. Voorhies and J. M. Scandura, "Determination of memory load in information processing," Problem Solving: a Structural/Process Approach with Instructional Implications, 1977.

[22] J. Smith-Spark, J. Fisk, A. Fawcett, and R. Nicolson, "Investigating the central executive in adult dyslexics: Evidence from phonological and visuospatial working memory performance," European Journal of Cognitive Psychology, vol. 15, no. 4, pp. 567587, 2003.

[23] C. Rosenquist, F. A. Conners, and B. RoskosEwoldsen, "Phonological and visuo-spatial working memory in individuals with intellectual disability," American Journal on Mental Retardation, vol. 108, no. 6, pp. 403413, 2003.

[24] R. Brunken, J. L. Plass, and D. Leutner, "Direct measurement of cognitive load in multimedia learning," Educational psychologist, vol. 38, no. 1, pp. 53-61, 2003.

[25] A. Baddeley, "Working memory and conscious awareness," in Theories of memory: Lawrence Erlbaum Associates, 1992, pp. 11-20.

[26] A. Baddeley, "Working memory: theories, models, and controversies," Annual review of psychology, vol. 63, pp. 1-29, 2012.

[27] G. Repovš and A. Baddeley, "The multicomponent model of working memory: explorations in experimental cognitive psychology," Neuroscience, vol. 139, no. 1, pp. 5-21, 2006.

[28] A. Baddeley, "The episodic buffer: a new component of working memory?," Trends in cognitive sciences, vol. 4, no. 11, pp. 417423, 2000.

[29] J. Sweller, "Cognitive load theory, learning difficulty, and instructional design," Learning and instruction, vol. 4, no. 4, pp. 295-312, 1994.

[30] J. Sweller, "Cognitive load theory: Recent theoretical advances," Cognitive load theory, vol. 1, pp. 29-30, 2010.

[31] F. Paas, A. Renkl, and J. Sweller, "Cognitive load theory and instructional design: Recent developments," Educational psychologist, vol. 38, no. 1, pp. 1-4, 2003.

[32] S. Kalyuga, "Cognitive load theory," in Managing Cognitive Load in Adaptive Multimedia Learning: IGI Global, 2009, pp. 34-57.

[33] R. Moreno and R. Mayer, "Interactive multimodal learning environments," Educational psychology review, vol. 19, no. 3, pp. 309-326, 2007.

[34] A. Baddeley, "Working memory: looking back and looking forward," Nature Reviews. Neuroscience, vol. 4, no. 10, p. 829, 2003.

[35] A. D. Baddeley and G. Hitch, "Working memory," Psychology of learning and motivation, vol. 8, pp. 47-89, 1974.

[36] A. Miyake and P. Shah, Models of working memory: Mechanisms of active maintenance and executive control. Cambridge University Press, 1999.

[37] D. Sridharan, D. J. Levitin, and V. Menon, "A critical role for the right fronto-insular cortex in switching between central-executive and default-mode networks," Proceedings of the National Academy of Sciences, vol. 105, no. 34, pp. 12569-12574, 2008.

[38] R. E. Mayer, "Multimedia learning," Psychology of learning and motivation, vol. 41, pp. 85-139, 2002.

[39] N. T. Sahin, S. Pinker, S. S. Cash, D. Schomer, and E. Halgren, "Sequential processing of lexical, grammatical, and phonological information within Broca's area," Science, vol. 326, no. 5951, pp. 445449, 2009.

[40] A. T. Sack et al., "Tracking the mind's image in the brain II: transcranial magnetic stimulation reveals parietal asymmetry in visuospatial imagery," Neuron, vol. 35, no. 1, pp. 195-204, 2002.

[41] P. Sauseng, W. Klimesch, M. Schabus, and M. Doppelmayr, "Fronto-parietal EEG coherence in theta and upper alpha reflect central executive functions of working 
memory," International Journal of Psychophysiology, vol. 57, no. 2, pp. 97-103, 2005.

[42] R. Cabeza and L. Nyberg, "Imaging cognition II: An empirical review of 275 PET and fMRI studies," Journal of cognitive neuroscience, vol. 12, no. 1, pp. 1-47, 2000.

[43] R. L. Klatzky, J. R. Marston, N. A. Giudice, R. G. Golledge, and J. M. Loomis, "Cognitive load of navigating without vision when guided by virtual sound versus spatial language," Journal of Experimental Psychology: Applied, vol. 12, no. 4, p. 223, 2006.

[44] Y.-J. Chang and T.-Y. Wang, "Comparing picture and video prompting in autonomous indoor wayfinding for individuals with cognitive impairments," Personal and Ubiquitous Computing, vol. 14, no. 8, pp. 737-747, 2010.

[45] E. Haapalainen, S. Kim, J. F. Forlizzi, and A. K. Dey, "Psycho-physiological measures for assessing cognitive load," in Proceedings of the 12th ACM international conference on Ubiquitous computing, 2010, pp. 301-310: ACM.

[46] D. Cyganski and R. J. Duckworth, "Search and rescue method and system," ed: Google Patents, 2016.

[47] I. F. Progri, W. R. Michalson, J. Wang, M. C. Bromberg, and J. Duckworth, "Requirements of a C-CDMA pseudolite indoor geolocation system," analysis, vol. 2, p. 12, 2017.

[48] T. Meilinger, M. Knauff, and H. H. Bülthoff, "Working memory in wayfinding - A dual task experiment in a virtual city," Cognitive Science, vol. 32, no. 4, pp. 755-770, 2008.

[49] S. G. Hart, "NASA-task load index (NASATLX); 20 years later," in Proceedings of the human factors and ergonomics society annual meeting, 2006, vol. 50, no. 9, pp. 904908: Sage Publications Sage CA: Los Angeles, CA.

[50] C. Hölscher and M. Brösamle, "Capturing indoor wayfinding strategies and differences in spatial knowledge with space syntax," in 6th International Space Syntax Symposium, 2007.

[51] S. Sternberg, "Memory-scanning: Mental processes revealed by reaction-time experiments," American scientist, vol. 57, no. 4, pp. 421-457, 1969.

[52] J. Jonides et al., "Verbal working memory load affects regional brain activation as measured by PET," Journal of cognitive neuroscience, vol. 9, no. 4, pp. 462-475, 1997.

[53] R. W. Engle, "Working memory capacity as executive attention," Current directions in psychological science, vol. 11, no. 1, pp. 1923, 2002.

[54] J. P. Tracy and M. J. Albers, "Measuring cognitive load to test the usability of web sites," in Annual Conference-society for technical communication, 2006, vol. 53, p. 256.

[55] D. G. Holmberg, W. D. Davis, S. J. Treado, and K. A. Reed, "Building Tactical Information System for Public Safety OfficialsIntelligent Building Response (iBR)," 2006.

[56] J. Averill, D. Holmberg, A. Vinh, and W. Davis, "Building Information Exchange for First Responders Workshop: Proceedings," NIST Technical Note.

[57] D. G. Holmberg, M. A. Raymond, and J. D. Averill, "Delivering building intelligence to first responders," NIST TN, vol. 1648, 2013.

[58] (2005). NEMA SB 30-2005: Fire Service Annunicator and Interface.

[59] E. Nunez, "Cognitive Assistant Systems for Emergency Response," 2017.

[60] B. D. Campbell, H. O. Mete, T. Furness, S. Weghorst, and Z. Zabinsky, "Emergency response planning and training through interactive simulation and visualization with decision support," in Technologies for Homeland Security, 2008 IEEE Conference on, 2008, pp. 176-180: IEEE.

[61] G.-J. M. Kruijff et al., "Experience in system design for human-robot teaming in urban search and rescue," in Field and Service Robotics, 2014, pp. 111-125: Springer.

[62] L. M. Reeves et al., "Guidelines for multimodal user interface design," Communications of the ACM, vol. 47 , no. 1 , pp. 57-59, 2004.

[63] FLAIM. (2018). FLAIM Trainer Official Website. Available: http://flaimtrainer.com/

[64] E2i. (2018). E2i Creative Studio Official Website. Available: http://e2i.ist.ucf.edu/

[65] ADMS-FIRE. (2018). ADMS-FIRE Training Official Website. Available: http://www.trainingfordisastermanagement.c om/products/adms-fire/

[66] N. J. Belkin and W. B. Croft, "Information filtering and information retrieval: Two sides of the same coin?," Communications of the $A C M$, vol. 35, no. 12, pp. 29-38, 1992. 